\title{
ASSOCIAC̣ÃO ENTRE LARVAS DE CHRYSOMYA MEGACEPHALA (FABRICIUS) E CHRYSOMYA ALBICEPS (WIEDEMANN), CHRYSOMYA MEGACEPHALA (FABRICIUS) E COCHLIOMYIA MACELLARIA (FABRICIUS) (CALLIPHORIDAE, DIPTERA) SOB CONDIC̣ÕES DE LABORATÓRIO ${ }^{1}$
}

\author{
Valéria Magalhães Aguiar-Coelho ${ }^{2}$ \\ Eliane M.V. Milward-de-Azevedo ${ }^{3}$
}

\begin{abstract}
ASSOCIATION BETWEEN CHRYSOMYA MEGACEPHALA (FABRICIUS) AND CHRYSOMYA ALBICEPS (WIEDEMANN), CHRYSOMYA MEGACEPHALA (FABRICIUS) AND

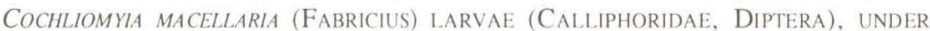
LABORATORY CONDITIONS. This paper intends to recognize some aspects of interspecific relationships between Chrysomya megacephala (Fabricius) and $C$. albiceps (Wiedemann) and between $C$. megacephala and Cochliomyia macellaria (Fabricius) larvae in laboratory. A diet consisting of decomposing horse flesh was used, and the relation of 1 larva/g diet was established. The development of the two species was done in pure and associated cultures. The association with Chrysomya albiceps influenced the post-embrionary development period and the weight of mature larvae of the $C$. megacephala, reducing their. Such weight decrease, along with the predation of larvae of $C$. megacephala larvae, yelded a decrease in survival in all of the stages. Larvae of this species showed an increase in its weight when associated with Cochliomyia macellaria. The native species responded to the association by reducing its body weigth and its viability. This association doesnt influence the duration of post-embrionary development.

KEY WORDS. Chrysomya, Cochliomyia macellaria, blowfly, interspecific relationship, larval development
\end{abstract}

Os califorídeos Chrysomya megacephala (Fabricius), Chrysomya albiceps (Wiedemann) e Cochliomyia macellaria (Fabricius, 1775) apresentam grande importância sócio-econômica, sendo considerados, por alguns autores, os dípteros vetores de microorganismos patogênicos mais perigosos (FURLANETTO et al. 1984; GreEnBerG 1988; Sulaiman et al. 1988, 1989). No entanto, a função ecológica destas espécies reside principalmente no fato de suas larvas desenvolverem-se ativamente em carcaças, atuando como decompositoras.

Vários autores tentaram explicar a coexistência de moscas varejeiras em recursos alimentares efêmeros. Segundo DENNO \& COTHRAN (1975) estes insetos

1) Pesquisa financiada pela FINEP/CNPq/PCTPA

2) Departamento de Patologia e Imunologia, Universidade de Nova Iguaçu. 26260-000 Nova Iguaçu, Rio de Janeiro, Brasil.

3) Departamento de Parasitologia, Universidade Federal Rural do Rio de Janeiro. 23851-970 Itaguaí, Rio de Janeiro, Brasil. Bolsista do CNPq. 
não exploram a carcaça como um recurso homogêneo; eles são, aparentemente, capazes de se especializarem em diferentes aspectos dietéticos, neste substrato. Sua distribuição é temporal. Tullis \& GOFF (1987) e GofF et al. (1988) sugeriram que cada espécie pode ter seu próprio nicho ou habitat dentro da carcaça, e exemplificaram que as larvas de Chrysomya rufifacies (Linnaeus) permanecem em massa frouxa, enquanto que as larvas de $C$. megacephala formam massas orgânicas em tecidos firmes situados abaixo de agrupamentos de $C$. rufifacies. Das teorias que tentam explicar a coexistência destas espécies, uma delas enfatiza a relatividade da habilidade competitiva e sugere que a especialização em diferentes partes do recurso alimentar ou a habilidade superior de dispersão, pode permitir a coexistência de espécies em um equilíbrio estável (MACARTHUR \& WILSON 1967; KNEIDEL 1984). Por outro lado, a reação heterotípica negativa entre califorídeos, envolvendo a participação de $C$. albiceps, foi experimentalmente detalhada por UllyetT (1950). Aguiar-Coelho et al. (no prelo) sugeriram que, embora a associação entre Cochliomyia macellaria e Chrysomya. albiceps seja mais deletéria para a espécie autóctone, o muscóide exótico também pode ser afetado. Contudo, pouco se conhece a respeito da interação entre C. megacephala, C. albiceps e Cochliomyia macellaria, e da influência que estas relações exercem sobre o desempenho específico destes insetos. Assim, o presente trabalho procurou, através da observação de algumas características biológicas e comportamentais monitoradas em culturas puras e mistas (processadas aos pares), verificar possíveis alterações determinadas pelas associações entre aquelas populações. Este estudo, parte de uma pesquisa em desenvolvimento, visa auxiliar a compreensão das relações inter-específicas existentes entre muscóides durante sua sucessão ecológica em carcaças e, conseqüentemente, subsidiar seu controle.

\section{MATERIAL E MÉTODOS}

Os procedimentos relativos ao estabelecimento e à manutenção dos estoques de Chrysomya megacephala, C. albiceps e Cochliomyia macellaria seguiram a metodologia descrita por MiLwARD-DE-AZEvedo et al. (no Prelo), QueIroz \& Milward-DE-AzEvedo (1991) e CunHA-E-Silva \& Milward-DE-AZEvedo (1994), respectivamente, efetuando-se reintroduções periódicas de espécimens nativos nas colônias. AgUiAR-COELHO et al. (no prelo) detalharam a metodologia empregada durante a condução das diferentes etapas experimentais.

Inicialmente, estudou-se a associação entre as larvas da sétima geração de Chrysomya megacephala e da quarta geração de Cochliomyia macellaria. Posteriormente, foram monitoradas as relações entre as larvas de Chrysomya megacephala e $C$. albiceps, oriundas da nona e quarta geração estoque, respectivamente. Utilizou-se a densidade de 1 larva/g de dieta à base de carne equina em decomposição. O experimento foi conduzido em câmara climatizada regulada a $30^{\circ} \mathrm{C}$ de temperatura, $60 \pm 10 \%$ de umidade relativa e 14 horas de fotofase.

Cada tratamento constou de quatro repetições. Nas culturas mistas, foram inoculadas 25 neolarvas de cada espécie, num total de 50 neolarvas, sobre $50 \mathrm{~g}$ de dieta. Nas culturas puras, respeitou-se a mesma densidade (1 larva/g de dieta), 
utilizando-se 50 neolarvas de cada espécie. As larvas que abandonaram a dieta foram individualizadas e pesadas. As observações foram diárias.

O efeito de combinação e o efeito de espécie foi analisado através do teste de Kruskal-Wallis (ZAR 1984). As estatísticas F calculadas foram consideradas significativas quando $\mathrm{p}<0,05$. Quando $0,05<\mathrm{p}<0,10$ foi referida tendência à significância. Os contrastes entre postos médios foram realizados com o cálculo da dms a $5 \%$.

\section{RESULTADOS E DISCUSSÃO}

Em culturas puras, a taxa de velocidade de desenvolvimento de larvas de Cochliomyia macellaria, na dieta, foi similar à apresentada por Chrysomya megacephala (Tab. I). Quase 100\% dos imaturos destas espécies abandonaram o substrato três dias após sua inoculação (Fig. 1). Entretanto, a emergência dos adultos das moscas-das-latrinas mostrou-se mais precoce (Tab. I, Fig. 2). As larvas das duas populações pesaram, em média, 69mg (Tab. II). CunHA-E-SILvA \& MILWARD-DE-AZEVEDO (1994) reportaram, para esta variável, valores acentuadamente inferiores, ao avaliarem o desempenho de Cochliomyia macellaria. As condições de armazenamento da carne que serviu como fonte de alimentação das larvas, no presente experimento, diferentes das estabelecidas por aqueles autores, deve ter influenciado a resposta, favorecendo a performance dos espécimens.

Tabela I. Duração do desenvolvimento pós-embrionário de Cochliomyia macellaria e Chrysomya megacephala isoladas e em associação, criadas em dieta à base de carne eqüina em decomposição, sob condições controladas $\left(30^{\circ} \mathrm{C}\right.$, UR. $60 \pm 10 \%$ e 14 h de fotofase).

\begin{tabular}{|c|c|c|c|c|}
\hline \multirow{3}{*}{ Espécies } & \multicolumn{4}{|c|}{ Duração (dias) } \\
\hline & Neolarvas ao abandono & Estágio larval & Estágio pupal & Neolarvas a adultos \\
\hline & $\bar{x}+5 \bar{x}$ & $\bar{x} \pm \overline{S x}$ & $\bar{x}+5 \bar{x}$ & $\bar{x} \pm S \bar{x}$ \\
\hline \multicolumn{5}{|l|}{ ISOLADA } \\
\hline C. macellaria & $3,08 \pm 0,01 \mathrm{Aa}$ & $4,01 \pm 0,01 \mathrm{Aa}$ & $4,19 \pm 0,09 \mathrm{Aa}$ & $8,19+0,09 \mathrm{Aa}$ \\
\hline C. megacephala & $3,06 \pm 0,44 \mathrm{Aa}$ & $4,06 \pm 0,04 \mathrm{Ab}$ & $3,68 \pm 0,07 \mathrm{Ab}$ & $7.73 \pm 0.09 \mathrm{Ab}$ \\
\hline \multicolumn{5}{|l|}{ ASSOCIADA } \\
\hline C. macellaria & 3,00 & 4,00 & $4,20 \pm 0,07 \mathrm{Aa}$ & $8,20+0,09 \mathrm{Aa}$ \\
\hline C. megacephala & $3,08+0,05 \mathrm{Ab}$ & $4,08 \pm 0,05 \mathrm{Ab}$ & $3,91 \pm 0,07 \mathrm{Bb}$ & $7,98 \pm 0,01 \mathrm{Bb}$ \\
\hline
\end{tabular}

1) As interações foram analisadas através do teste $F$, ao nível de $5 \%$ de probabilidade.

2) Médias seguidas pelas mesmas letras (efeito de combinação: letra maiúscula; efeito de espécie: letra minúscula), não diferem entre si pelo método das diferenças mínimas significativas (dms).

A velocidade de desenvolvimento pós-embrionário da espécie autóctone não foi influenciada pela sua associação com Chrysomya megacephala (Tab. I). O ritmo de abandono das larvas de Cochliomyia macellaria da dieta também não foi alterado, enquanto cerca de $30 \%$ das larvas de Chrysomya megacephala, neste tratamento, abandonaram o substrato alimentar mais tardiamente (Fig. 1). A 


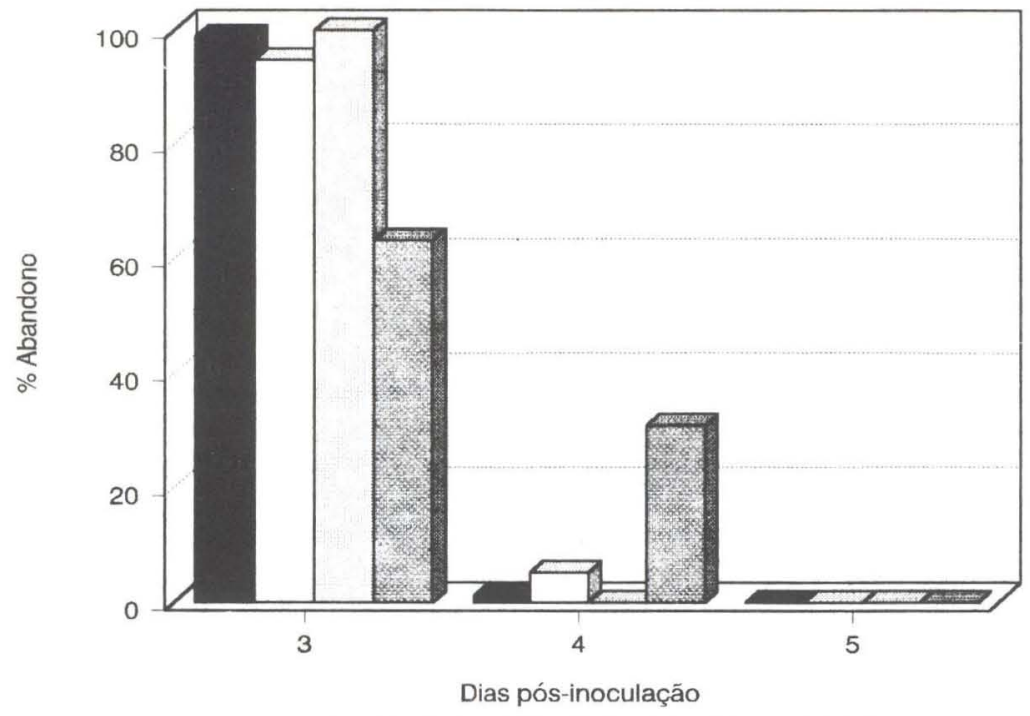

$\square$ Co. macellaria (I) $\square$ Ch. megacephala (I) $\square$ Co. macellaria (A) BCh. megacephala (A)

Fig. 1. Ritmo de abandono de larvas de Cochliomyia macellaria e Chrysomya megacephala de uma dieta à base de carne eqüina em decomposição, em culturas isoladas (I) e associadas (A), sob condições controladas $\left(30^{\circ} \mathrm{C}\right.$, UR. $60 \pm 10 \%$ e $14 \mathrm{~h}$ de fotofase).

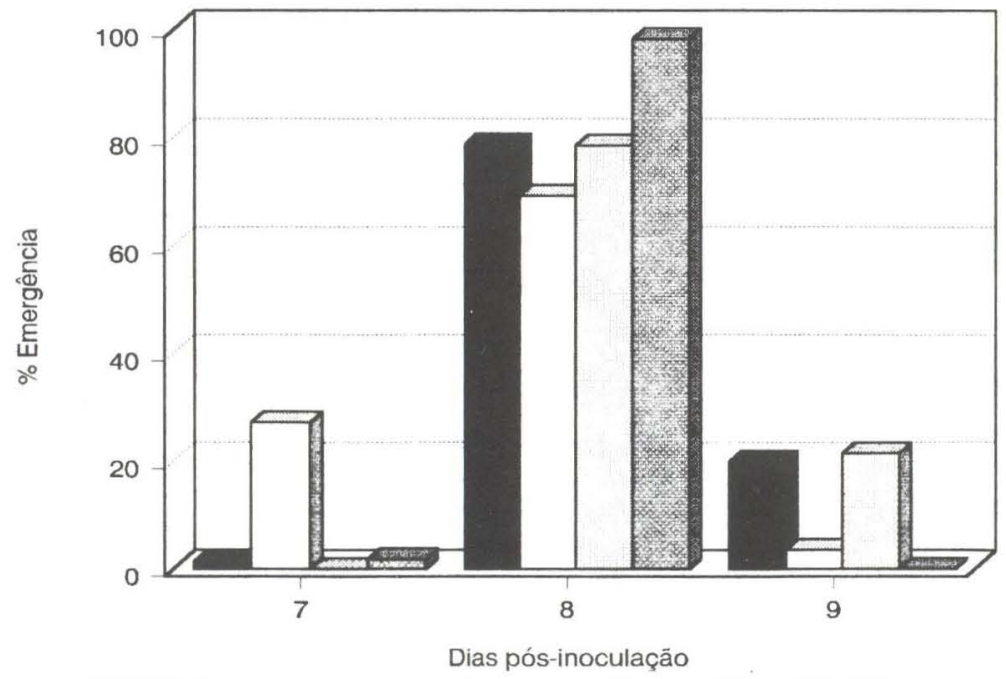

Co. macellaria (I) $\square$ Ch. megacephala (I) $\square$ Co. macellaria (A) $\square$ Ch. megacephala (A)

Fig. 2. Ritmo de emergência dos adultos de Cochliomyia macellaria e Chrysomya megacephala criadas em dieta à base de carne eqüina em decomposição, em culturas isolads (I) e associadas (A), sob condições controladas $\left(30^{\circ} \mathrm{C}\right.$, UR. $60 \pm 10 \%$ e $14 \mathrm{~h}$ de fotofase).

Revta bras. Zool. 12 (4): 991 - 1000, 1995 
associação provocou uma redução significativa do peso médio de larvas de Cochliomyia macellaria; as larvas de Chrysomya megacephala, por outro lado, apresentaram-se mais pesadas (Tab. II). Essas diferenças, entretanto, não determinaram alterações significativas na taxa de sobrevivência larval, nas duas populações; entretanto, ocorreu uma redução significativa no percentual de emergência de adultos de Cochliomyia macellaria (Tab. II).

Tabela II. Peso de larvas que abandonaram a dieta e taxas de sobrevivência de adultos de Cochliomyia macellaria e Chrysomya megacephala isoladas e em associação, criadas em dieta à base de carne eqüina em decomposição, sob condições controladas $\left(30^{\circ} \mathrm{C}, \mathrm{UR}\right.$. $60 \pm 10 \%$ e $14 \mathrm{~h}$ de fotofase).

\begin{tabular}{|c|c|c|c|c|}
\hline \multirow{2}{*}{ Espécies } & \multirow{2}{*}{ Peso de larvas (mg) } & \multicolumn{3}{|c|}{ Sobrevivência (\%) } \\
\hline & & Larval & Pupal & Neolarvas a adultos \\
\hline \multicolumn{5}{|l|}{ ISOLADA } \\
\hline C. megacephala & $68,92 \pm 0,63 \mathrm{Aa}$ & $92,00 \mathrm{Aa}$ & $86,96 \mathrm{Aa}$ & $80,00 \mathrm{Aa}$ \\
\hline C. albiceps & $68,87 \pm 0,45 \mathrm{Aa}$ & $78,00 \mathrm{Ab}$ & $95,55 \mathrm{Ab}$ & $73,50 \mathrm{Ab}$ \\
\hline \multicolumn{5}{|l|}{ ASSOCIADA } \\
\hline C. megacephala & $66,89 \pm 0,34 \mathrm{Ba}$ & $90,67 \mathrm{Aa}$ & $80,81 \mathrm{Ba}$ & $73,33 \mathrm{Ba}$ \\
\hline C. albiceps & $75,53+0,40 \mathrm{Bb}$ & $81,33 \mathrm{Ab}$ & $88,37 \mathrm{Bb}$ & $72,00 \mathrm{Aa}$ \\
\hline
\end{tabular}

1) As interações foram analisadas através do teste $F$, ao nivel de $5 \%$ de probabilidade.

2) Médias seguidas pelas mesmas letras (efeito de combinação: letra maiúscula; efeito de espécie: letra minúscula), não diferem entre si pelo método das diferenças mínimas significativas (dms).

Em culturas puras, a velocidade de desenvolvimento das fases imaturas de Chrysomya megacephala foi menor do que a observada para Chrysomya albiceps. A análise do efeito de espécie, apontou uma diferença significativa (Tab. III). LEVOT et al. (1979), ao compararem o desenvolvimento pós-embrionário de algumas espécies de califorídeos, entre eles, Chrysomya megacephala e $C$. rufifacies (espécie de comportamento similar a $C$. albiceps), observaram semelhante resultado. Nestas culturas, o peso médio de larvas de C. albiceps, após o abandono da dieta $(81,08 \mathrm{mg}$, em média), foi significativamente superior ao apresentado pelas larvas de C. megacephala (aproximadamente, 69mg) (Tab. IV). Registrou-se, também, um percentual acentuadamente maior de adultos de $C$. albiceps, nestas criações (Tab. IV). Os resultados obtidos estão de acordo com os mencionados por QUEIROZ \& MILWARD-DE-AZEVEDO (1991) e MILWARD-DEAzEvedo et al. (no Prelo) que trabalharam com culturas puras de C. albiceps e C. megacephala, respectivamente, e também assumiram a utilização de densidades independentes.

A associação entre as larvas de $C$. megacephala e $C$. albiceps influenciou a duração de todos os estágios de desenvolvimento pós-embrionário de $C$. megacephala, diminuindo-o significativamente (Tab. III). Observou-se um acentuado percentual de abandono de larvas da dieta no terceiro dia após a inoculação dos espécimens em cultura mista (Fig. 3). Cerca de 95\% dos adultos dessa espécie emergiram no oitavo dia, neste tratamento (Fig. 4). 


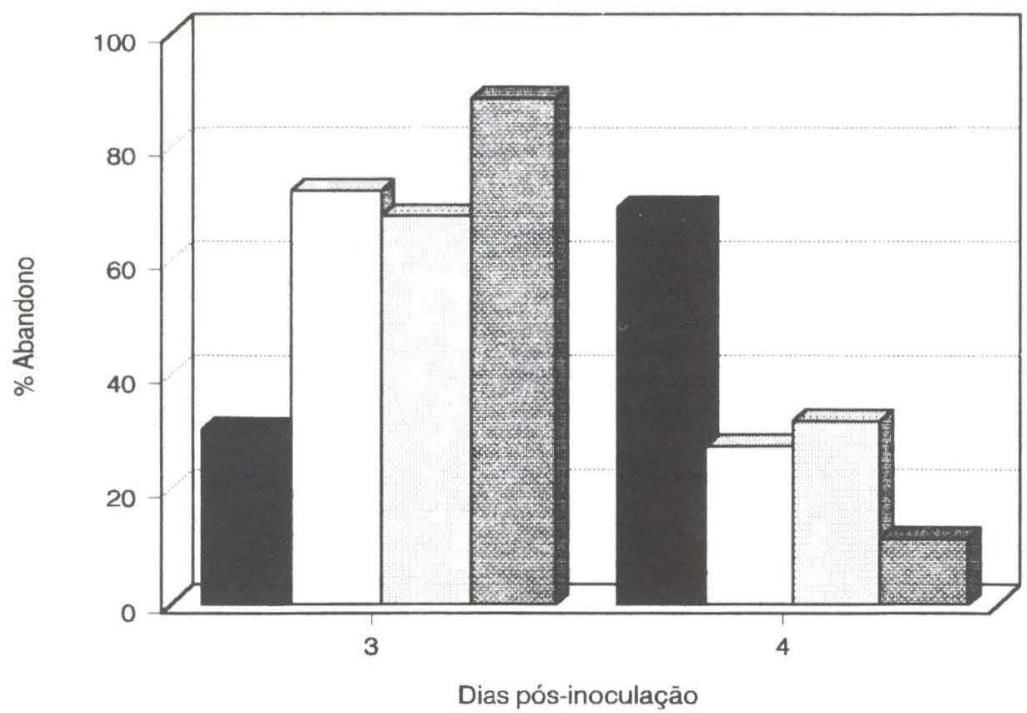

Ch. albiceps (I) $\square$ Ch. megacephala (I) $\square$ Ch. albiceps (A) ⿴囗玉 Ch. megacephala (A)

Fig. 3. Ritmo de abandono de larvas de Chrysomya megacephala e C. albiceps de uma dieta à base de carne eqüina em decomposição, em culturas isolads (I) e associadas (A), sob condições controladas $\left(30^{\circ} \mathrm{C}\right.$, UR. $60 \pm 10 \%$ e $14 \mathrm{~h}$ de fotofase).

O abandono de larvas de $C$. albiceps da dieta foi acentuadamente mais precoce em cultura mista (terceiro dia, respectivamente) (Fig. 3). Para este muscóide, apenas a duração do estágio pupal não foi significativamente reduzida pela associação (Tab. III). Estes resultados refletiram-se sobre o ritmo de emergência dos adultos (Fig. 4).

A associação com $C$. albiceps provavelmente contribuiu para o abandono precoce das larvas de $C$. megacephala da dieta e a redução significativa de seu peso (Tab. IV). Sugere-se que esta redução de peso esteja também, entre outros fatores, relacionada a fatores bióticos introduzidos e/ou provocados pela espécie associada, alterando a microbiota da dieta. Esta alteração provocaria mudanças na textura, no $\mathrm{pH}$ e na composição da dieta, fatores que influenciam diretamente o consumo e utilização do alimento pelos insetos (SLANSKY \& SCRIBER 1985). Para as larvas de $C$. albiceps, a redução de peso observada não foi significativa (Tab. IV).

A predação de larvas de $C$. megacephala por larvas de $C$. albiceps foi evidenciada fora da dieta alimentar. O comportamento predatório de $C$. albiceps, que variou de 15,8 a $85,0 \%$ entre as repetições, aliado ao decréscimo do peso de larvas maduras de $C$. megacephala, produziu reduções significativas nas taxas de sobrevivência do estágio larval e do período de neolarvas a adultos da mosca-daslatrinas (Tab. IV). Embora a taxa de sobrevivência total de C. megacephala não tenha sido tão drasticamente afetada pela associação com $C$. albiceps quanto à 
reconhecida para Cochliomyia macellaria (AGUIAR-COELHO et al. no prelo), ela alcançou um índice de redução de $40 \%$. A redução sofrida pela espécie autóctone, mantida sob condições experimentais similares, foi de $66 \%$, segundo AGUIARCoElHo et al. (no prelo). Por outro lado, a taxa de sobrevivência do estágio larval de Chrysomya albiceps também foi significativamente reduzida, provocando, nesta espécie, uma queda acentuada no percentual de adultos formados (Tab. IV).

Tabela III. Duração do desenvolvimento pós-embrionário de Chrysomya megacephala e C. albiceps isoladas e em associação, criadas em dieta à base de carne eqüina em decomposição, sob condições controladas $\left(30^{\circ} \mathrm{C}\right.$, UR. $60 \pm 10 \%$ e $14 \mathrm{~h}$ de fotofase).

\begin{tabular}{|c|c|c|c|c|}
\hline \multirow{3}{*}{ Espécies } & \multicolumn{4}{|c|}{ Duraçāo (dias) } \\
\hline & Neolarvas ao abandono & Estágio larval & Estágio pupal & Neolarvas a adultos \\
\hline & $\bar{x} \pm S \bar{x}$ & $\bar{x}+5 \bar{x}$ & $\bar{x}+5 \bar{x}$ & $\bar{x}+5 \bar{x}$ \\
\hline \multicolumn{5}{|l|}{ ISOLADA } \\
\hline C. megacephala & $3,28 \pm 0,10 \mathrm{Aa}$ & $4,26 \pm 0,11 \mathrm{Aa}$ & $4,08 \pm 0,06 \mathrm{Aa}$ & $8,29 \pm 0,09 \mathrm{Aa}$ \\
\hline C. albiceps & $3,68 \pm 0,25 \mathrm{Ab}$ & $4,70 \pm 0,23 \mathrm{Ab}$ & $4,29 \pm 0,23 \mathrm{Ab}$ & 9.00 \\
\hline \multicolumn{5}{|l|}{ ASSOCIADA } \\
\hline C. megacephala & $3,17 \pm 0,19 \mathrm{Ba}$ & $4,08+0,09 \mathrm{Ba}$ & 4,00 & $8,05+0,06 \mathrm{Ba}$ \\
\hline C. albiceps & $3,35 \pm 0,23$ & $4,33 \pm 0,23 \mathrm{Bb}$ & $4,51 \pm 0,16 \mathrm{Ab}$ & $8,85+0,09 \mathrm{Bb}$ \\
\hline
\end{tabular}

1) As interações foram analisadas através do teste $F$, ao nível de $5 \%$ de probabilidade.

2) Médias seguidas pelas mesmas letras (efeito de combinação: letra maiúscula; efeito de espécie: letra minúscula), não diferem entre si pelo método das diferenças mínimas significativas (dms).

Tabela IV. Peso de larvas que abandonaram a dieta e taxas de sobrevivência de adultos de Chrysomya megacephala e C. albiceps isoladas e em associação, criadas em dieta à base de carne eqüina em decomposição, sob condições controladas $130^{\circ} \mathrm{C}$, UR, $60 \pm 10 \%$ e $14 \mathrm{~h}$ de fotofase).

\begin{tabular}{|c|c|c|c|c|}
\hline \multirow{2}{*}{ Espécies } & \multirow{2}{*}{$\frac{\text { Peso de larvas (mg) }}{\bar{x} \pm 5 \bar{x}}$} & \multicolumn{3}{|c|}{ Sobrevivência (\%) } \\
\hline & & Larval & Pupal & Neolarvas a adultos \\
\hline \multicolumn{5}{|l|}{ ISOLADA } \\
\hline C. macellaria & $68,82 \pm 0,46 \mathrm{Aa}$ & $81,50 \mathrm{Aa}$ & $90,31 \mathrm{Aa}$ & $73,50 \mathrm{Aa}$ \\
\hline C. megacephala & $81.08 \pm 5.91 \mathrm{Ab}$ & $96.50 \mathrm{Ab}$ & $91.25 \mathrm{Aa}$ & $88,00 \mathrm{Ab}$ \\
\hline \multicolumn{5}{|l|}{ ASSOCIADA } \\
\hline C. macellaria & $49,65 \pm 1,38 \mathrm{Ba}$ & $35,00 \mathrm{Ba}$ & $83,97 \mathrm{Aa}$ & $33,00 \mathrm{Ba}$ \\
\hline c. megacephala & $75,11 \pm 4,00 \mathrm{Ab}$ & $78,00 \mathrm{Bb}$ & $83,47 \mathrm{Aa}$ & $65,00 \mathrm{Bb}$ \\
\hline
\end{tabular}

1) As interações foram analisadas através do teste $F$, ao nivel de $5 \%$ de probabilidade.

2) Médias seguidas pelas mesmas letras (efeito de combinação: letra maiúscula; efeito de espécie: letra minúscula), não diferem entre si pelo método das diferenças mínimas significativas (dms).

Nas culturas mistas, as larvas foram individualizadas logo após o abandono espontâneo e/ou estratégico da dieta. Assim, o comportamento predatório desenvolvido por $C$. albiceps só foi permitido até este procedimento. Na natureza, a 


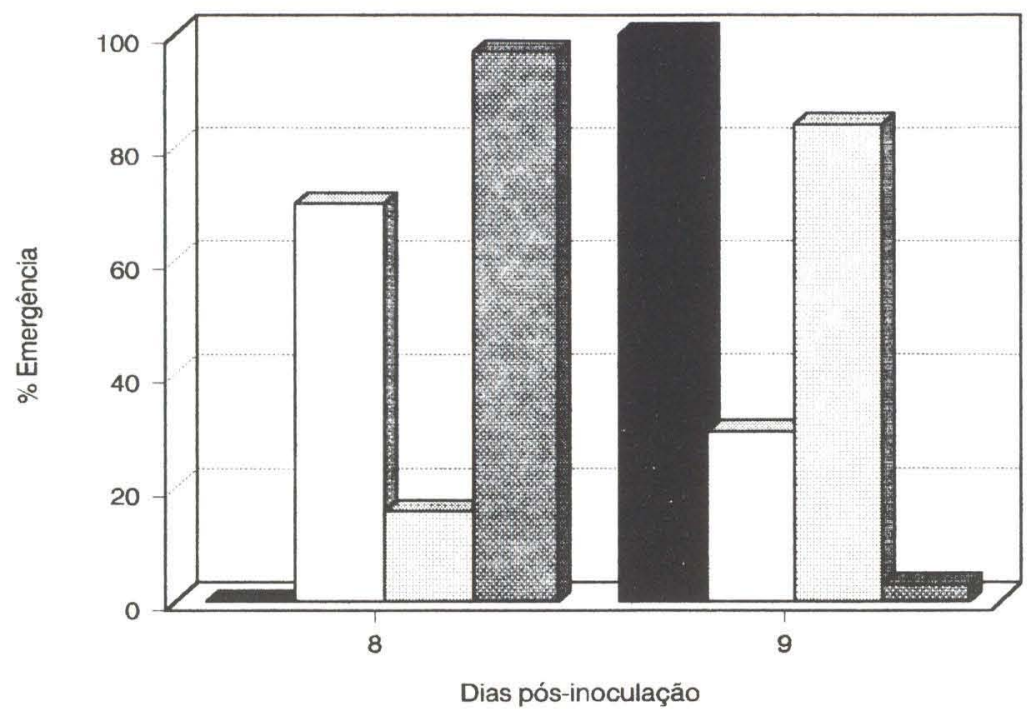

Ch. albiceps (I) $\square$ Ch. megacephala (I) WCh. albiceps (A) .

Fig. 4. Ritmo de emergência dos adultos de Chrysomya megacephala e C. albiceps criadas em dieta à base de carne eqüina em decomposição, em culturas isolads (I) e associadas (A), sob condições controladas $\left(30^{\circ} \mathrm{C}\right.$, UR. $60 \pm 10 \%$ e $14 \mathrm{~h}$ de fotofase).

grande habilidade migratória de espécies competitivamente inferiores tem possibilitado a capacidade de sobrevivência destas espécies quando coexistem na mesma fonte alimentar (BEAVER 1977). No presente trabalho, as baixas taxas de sobrevivência obtidas para $C$. megacephala, retroalimentando discussões sugeridas em experimentos anteriormente conduzidos por AGUIAR-CoElHo et al. (no prelo) (onde os autores analisaram a associação existente entre Cochliomyia macellaria e Chrysomya albiceps), talvez se devam ao confinamento dos espécimens em uma área restrita. GREENBERG (1990) constataram, por exemplo, que larvas de Cochliomyia macellaria podem se dispersar até $2,4 \mathrm{~m}$ além do sítio de alimentação. LAAKE et al. (1936) descreveram o comportamento de enterramento caracterizado por esta espécie. Estes procedimentos incluem relevantes mecanismos de defesa contra potenciais predadores e/ou parasitóides, na natureza. O reconhecimento dos mecanismos de defesa utilizados por espécies estrategistas r, como Chrysomya megacephala, compromete, sem dúvida, a compreensão da dinâmica sugerida por estes decompositores, impondo a necessidade de investigações mais detalhadas.

AGRAdeCIMENTOS. Ao Prof. Paulo Roberto Curi, da Universidade Estadual Paulista, Botucatu (São Paulo), pela execução da análise estatística. A Coordenadoria de Aperfeiçoamento de Pessoal de Nível Superior (CAPES) pelo suporte financeiro dotado à primeira autora. 


\section{REFERÊNCIAS BIBLIOGRÁFICAS}

Aguiar-Coelho, V.M.; M.M.C. Queiroz \& E.M.V. Milward-DE-AZEvedo. (no prelo). Associações entre larvas de Cochliomyia macellaria (Fabricius) e Chrysomya albiceps (Wiedemann) (Diptera, Calliphoridae), sob condições de laboratório. Revta bras. Zool. .

BEAvER, R.A. 1977. Non-equilibrium "island" communities: Diptera breeding in dead snails. Jour. Anim. Ecol. 46: 783-798.

CunHA-E-Silva, S.L. \& E.M.V. Milward-DE-AZEvedo. 1994. Estudo comparado do desenvolvimento pós-embrionário de Cochliomyia_macellaria (Fabricius) (Diptera, Calliphoridae) em duas dietas à base de carne, em laboratório. Revta bras. Zool. 11: 649-658.

DENNO, R.F. \& W.R. COTHRAN. 1975. Niche Relationships of a guild of necrophagous flies. Ann. Entomol. Soc. Amer. 68: 741-754.

FurlanetTo, S.M.P.; M.L.C. Campos; C.M. Harsi; G.M. Buralli \& G.K. ISHIHATA. 1984. Microorganismos enteropatogênicos em moscas africanas pertencentes ao gênero Chrysomya (Diptera, Calliphoridae) no Brasil. Rev. Microbiol. 15: 170-174.

GofF, M.L.; A.J. OMORI \& K. GunATIKLAKE. 1988. Estimation of postmortem interval by arthropod succession: three case studies from the Hawaiian Islands.

Amer. Jour. Forensic Med. Pathol. 9: 220-225.

GreEnBerg, B. 1988. Chrysomya megacephala (F.) (Diptera: Calliphoridae) collected in North America and notes on Chrysomya species present in the New World. Jour. Med. Entomol. 25: 199-200.

1990. Nocturnal oviposition behavior of blow flies (Diptera: Calliphoridae). Jour. Med. Entomol. 27: 807-810.

KNEIDEL, K.A. 1984. Competition and disturbance in communities of carrionbreeding diptera. Jour. Anim. Ecol. 53: 849-865.

LAake, E.W.; E.C. Cushing \& H.E. PARish. 1936. Biology of the primary screwworm fly, Cochliomyia americana, and a comparison of its stages with those of C. macellaria. USDA Tech. Bull., Washington, 500: 1-24.

LEVOT, G.W.; K.R. BROWN \& E. SHIPP. 1979. Larval growth of some calliphorid and sarcophagid Diptera. Bull. Entomol. Res. 69: 469-475.

MACARTHUR, R.H. \& E.O. WILSON. 1967. The theory of island biogeography. Princeton, Princeton Univ. Press, 203p.

Milward-de-Azevedo, E.M.V.; J.D.A. Herzog; M.A.S. Freitas \& E.H.S. FARIA. (no prelo). Desenvolvimento ontogenético, potencial reprodutivo e longevidade de Chrysomya megacephala (Fabricius) (Diptera: Calliphoridae) em condições de laboratório. Revta bras. Ent. 39 (3).

Queiroz, M.M.C. \& E.M.V. Milward-DE-AzEvedo. 1991. Técnicas de criação e alguns aspectos da biologia de Chrysomya albiceps (Wiedemann) (Diptera, Calliphoridae), em condições de laboratório. Revta bras. Zool. 8: 75-84.

SlanSky, JR. F. \& M. SCriber. 1985. Food consumption and utilization, 87-163. In: M.G.A. Kerkut \& L.J. Gilbert Comprehensive Insect Physiology, 
Biochemistry and Pharmacology. Oxford, Pergamon Press, 162p.

Sulaiman, S.; A.L. Sohadi; H. Yunus \& R. Iberahim. 1988. The role of some cyclorrhaphan flies as carriers of human helminths in Malaysia. Med. Vet. Entomol. 1: 1-6.

Sulaiman, S.; A.R. SOHADI \& J. JefFery. 1989. Human helminth parasite birdens on cyclorrhaphan flies (Diptera) trapped at an aboriginal settlement in Malaysia. Bull. Entomol. Res. 79: 625-629.

Tullis, K. \& M.L. GofF. 1987. Arthropod succession in exposed carrion in a tropical rainforest on O'ahu Island, Hawaií. Jour. Med. Entomol. 24: 332-338.

UlLyeTT, G.C. 1950. Competition for food and allied phenomena in sheepblowfly populations. Philos. Trans. R. Soc. Lond. Biol. Sci. 234: 77-174.

ZAR, J.H. 1984. Biostatistical Analysis. Englewood Cliffs, Prentice-Hall International Editions, $2^{\text {nd }}$ ed., 718 p. 